\title{
Clinical Trial Simulation in Drug Development
}

\author{
Pascal Girard, ${ }^{1}$ Michel Cucherat, ${ }^{2}$ David Guez ${ }^{3}$ and the Participants in Round Table \\ No. 2, Giens XIX \\ 1 INSERM et EA 3738 Ciblage Thérapeutique en Oncologie, Faculté de Médecine Lyon-Sud, Lyon, France \\ 2 Hôpital Laennec, Pharmacologie EA 643, Lyon, France \\ 3 Laboratoires Servier, Neuilly-sur-Seine, France
}

\begin{abstract}
The simulation of therapeutic models and clinical trial simulation have recently attracted attention as emerging techniques for developing new active molecules and the exploration of possible clinical trial results. Such approaches have benefited from fundamental progress in the development of 'in silico' models, as well as progress in nonlinear mixed-effect pharmacokinetic-pharmacodynamic models. Mixing the two approaches allows simulation of 'virtual' patients, who receive virtual treatments or placebo. These have various uses, such as proof of concept, decision analysis or experimental design optimisation. Also, the effect of departures from protocol on clinical trial results can easily be evaluated by the use of simulation. This technique is now implemented by the pharmaceutical industry for optimising phase II and III experimental designs when a good biomarker or a clinical outcome model is available, but the use of an in silico therapeutic model as a proof of concept is only just beginning. In order to see such methodologies used more widely in drug development, multidisciplinary efforts need to be initiated, new modelling and simulation tools developed, and sound modelling and simulation practice documents need to be adopted. A reduction in the number of failed clinical development projects, the number of negative phase II and III clinical trials, or in just their cost and duration, are among the expected benefits of modelling and simulation in clinical drug development.
\end{abstract}

\section{Introduction}

The topic of this round table was simulation of clinical trials, and has recently attracted attention as an emerging technique for the synthesis of knowledge and the exploration of possible clinical trial results on the basis of a mathematical/stochastic model of the drug action and disease process combined with a model of the trial. ${ }^{[1-7]}$ This approach benefits from fundamental progress in the development of 'in silico' models such as the Physiome project, ${ }^{[8]}$ as well as progress in nonlinear mixed-effect models, which are generally further removed from the underlying physiology, but allow the inclusion of different sources of variability (between and within patients) and uncertainty. ${ }^{[9]}$ Mixing the two approaches allows simulation of 'virtual' patients, which has various uses, such as proof of concept, decision analysis or experimental design optimisation. The word 'virtual' is used in quotation marks since, as discussed during the round table, this is still a science-fiction high-tech concept. Nevertheless, although this approach was initiated in clinical pharmacology, it is already widely used in other industries, e.g., in automobile design or aeronautics, where flight simulators are now widely used both for aircraft design or pilot training. ${ }^{[10]}$ The round table has explored the different concepts and definitions of clinical trial simulation (CTS), its advantages and limitations, as well as its area of application and actual penetration in both the pharmaceutical and academic worlds in France.

\section{Definitions}

\subsection{General Definitions}

Before entering into any discussion of CTS, some definitions/clarifications are needed:

$\dagger$ For a list of participants, please see the end of the article. 
- Model: a mathematical representation of the behaviour of a system.

- Simulation: a study of the behaviour of a system by implementing a computer-based mathematical model.

- Clinical trial simulation: a study of selected outcomes of a clinical trial, defined by its experimental design, by implementing a computer-based mathematical model and design. Models can essentially be of two types, descriptive or mechanistic, with some subtle differences between the two. ${ }^{[11,12]}$ The use of mechanistic models allows the definition of a second type of simulation, i.e., simulation of a therapeutic model that makes use of mechanistic models. In this article, we describe in more detail the essential characteristics of these two types of models and simulation concepts.

\subsection{Descriptive Models}

Descriptive models are mathematical representations of the behaviour of a system where the only requirements are to fit the data and present a data summary using a restricted set of parameters. Such models totally ignore the mechanisms and underlying physiology of the biological phenomenon they are describing. Typical examples are the polynomial model or the spline models, which can fit any type of nonlinear curve. ${ }^{[13]}$ The principal advantages of descriptive models are their flexibility (they can adjust to any type of curve), their generic nature, simplicity and ease of implementation. Their main drawbacks are, firstly, poor interpolative/extrapolative properties since, outside of the range of observed concentrations and/or doses used for establishing the model, such models have no, or very limited, predictive capabilities; and, secondly, the fact that they provide no physiological interpretations of their parameters. However, some of these descriptive models offer access to some physiological interpretations of their parameters. Pharmacokinetic (PK) models to some extent belong in this category in the sense that they are mostly presented as a sum of exponentials meant to represent compartments with no physiological meaning at all. However, they allow a meaningful physiological parameter - clearance - to be computed. Similarly, the hyperbolic parameterisation of the $\mathrm{E}_{\max }$ (maximum effect) model derives from the in vitro receptor theory, which is no longer valid when considering a response measured in a complete living organism. However, it allows computation of the concentration producing a $50 \%$ change in the maximum effect, which relates directly to drug potency defined as the ratio between the association and dissociation rate constant between drug and receptor. ${ }^{[14,15]}$ This is true for hyperbolic models used in direct-action as well as indirect-action models. ${ }^{[11,16]}$ In this sense, compartmental as well as $\mathrm{E}_{\max }$ models lie between descriptive and mechanistic models. However, their performance is poor in terms of extrapolations, which can only be achieved through mechanistic models.

\subsection{Mechanistic Models}

Mechanistic models refer to sets of equations, each one describing a physiological process, either at the level of a cell or an organ. These models are also known as physiological models or phenomenological models in the sense that each equation describes one physiological phenomenon. Classical examples are the physiologically based PK models (PBPK) that model flows and exchanges between and within different organs. ${ }^{[12]}$ Similarly, these types of models have been developed for many pharmacodynamic (PD) processes, in particular those involving neurotransmitters, hormones or any other endogenous molecules. ${ }^{[11,17-}$ ${ }^{20]}$ Most of the time, such models necessitate the use of ordinary or stochastic differential equations, each set of equations describing transfer rates between organs and/or cells, according to linear or nonlinear processes in the derivative space. It is worth noticing that the closer the equations are to the physiological model, e.g., at a cellular level, the more data will be needed to identify the model parameters. For example, PBPK models need, to be identifiable, concentration data derived from the different organs, which for the most part means sacrifice of the organism and thus limits PBPK to study in small mammals. One of the highest levels of integration for those models is provided by the Physiome project, which "is a multicentric integrated program to design, develop, implement, test and document, archive and disseminate quantitative information, and integrative models of the functional behaviour of molecules, organelles, cells, tissues, organs, and intact organisms from bacteria to man". ${ }^{[8]}$ At this level of complexity, it is misleading to assume that the model can be identified in a representative sample of patients, since the data are too complex and too expensive to gather. Consequently, when it is necessary to use such models in patient samples, choices have to be made between complexity and simplification. One solution is to mix physiological and nonphysiological nonparametric models, or to identify part of the model using available data and to fix other mechanistic parts that are not identifiable using prior information. ${ }^{[6,21]}$

\subsubsection{Convergence Between the Two Types of Models}

At present, the two types of models are converging; PK-PD models tend to be increasingly complex, integrating physiopathological knowledge, or part of the mechanistic model tends to be simplified so that it can be used in routine simulations. Within this convergence, the mathematical models used to fit the data 
are constructed on the basis of physiopathological mechanisms, and not only because of considerations of goodness of fit.

\subsection{Simulation of the Therapeutic Model}

The sum of knowledge regarding the fundamental physiopathological and physiological mechanisms involved in an illness continuously increases, as does knowledge on the mechanisms of action of various treatments. Given the experimental process, this knowledge addresses only isolated elements of a system: genome, cell, tissue, and organ. Innovation and research necessitate an integration of all this knowledge into a global schema - the therapeutic model. Until now, this was performed in a discursive fashion. The absence of formalism in this approach does not allow simultaneous integration of all the dimensions of complexity because of their mono- or pauci-factorial and, overall, nonquantitative nature. This level of complexity prevents all the complex consequences of a treatment from being taken into account. Examples where this approach has failed are numerous, e.g., the use of antiarrhythmic drugs during acute myocardial infarction, phosphodiesterase inhibitors, etc. Most of the time, integration is limited, focuses only on the treatment benefits and partially neglects metabolism and harmful adverse effects. The limitations in the methods used in building therapeutic models mean that large and costly clinical trials are required because of weak assumptions and the fact that available global knowledge is not taken into account.

\subsection{Clinical Trial Simulation and 'Virtual' Patients}

To perform CTS, several types of models are needed in order to describe the disease and the treatment: study design, dosage regimens, population pharmacokinetics and pharmacodynamics, disease progression, placebo response, compliance patterns, dropout rates, study endpoints, sample schedules and approaches to statistical analysis. ${ }^{[22]}$ Figure 1 exemplifies some of the complex interactions that can be found between drug-disease and execution models. These models need to be applied to the socalled 'virtual' patients. This is something of an abuse of the term, since those patients are defined by few covariates (age, gender, weight, genotype or phenotype and biomarkers), which do not explain all the complex biological interactions between the body, the disease and the drug. These covariates can be generated either by a model or resampled from an existing database. For example, gender can easily be simulated from a binomial distribution and age from a log-normal truncated distribution. However, when introducing physiopathological covariates, one has to take into account correlations between the different covariates. Typically, height and size are correlated with gender, and hepatic and renal function are mainly correlated with age. In addition, the latter covariates can have multimodal distributions depending on genetic differences. The problem is even more complex when the covariate changes over time (e.g. a bone marker in osteoporosis), which requires both a longitudinal model to describe those changes in addition to a correlation model. ${ }^{[23]}$ The more covariates that are introduced, the more realistic the 'virtual' patient should be and the more complex the 'virtual' patient model becomes, although there is still a danger of simulating highly improbable patients because all those complex correlations or changes are ignored. An alternative to simulating 'virtual' patients is to use epidemiological databases and to resample real patients from them. An example is given in a CTS project for angina pectoris where, rather than simulating individual heartrate profiles that were predictive of angina pectoris risk, the heart rates were resampled from the OCTAVE (Optimisation du Contrôle de la Tension Artérielle, Valeur de l'Enregistrement sur $24 \mathrm{~h}$ ) database. ${ }^{[6]}$ This leads to the creation of 'hybrid' patients, where part of the data characterising them is resampled from existing databases, and part is simulated by a model.

\section{Perimeters of the Use of Modelling and Simulation (M\&S)}

\subsection{Objectives and Benefits}

Modelling and Simulation (M\&S) is a generic tool, which can be used at different levels of drug development: (i) in preclinical studies (structure-activity relationships as well as toxicity studies), as a proof of concept using mechanistic models for checking therapeutic assumptions; (ii) in extrapolating from animal to man using allometric models and pre-defining doses in phase I; and (iii) in phase II and III studies for protocol optimisation and decision analysis (sections 3.3 and 3.4). The main objectives of $M \& S$ are to offer a quantitative basis for decision analysis. Those decisions made on the basis of $M \& S$ range from improvement in clinical trial design to rejection of valueless studies or studies that could potentially fail, and from completing a study within a shorter time-frame and/or more cheaply to stopping the development of a drug.

A secondary benefit of $M \& S$ is to bring together people from different areas in drug development (pharmacologists, clinicians, pharmacometricians, statisticians, drug developers etc.), in order to facilitate and promote their collaboration by suggesting they share knowledge through modelling, mathematically formalising assumptions, and by a better understanding of all the dimensions of the product under development. In this sense, M\&S and CTS 


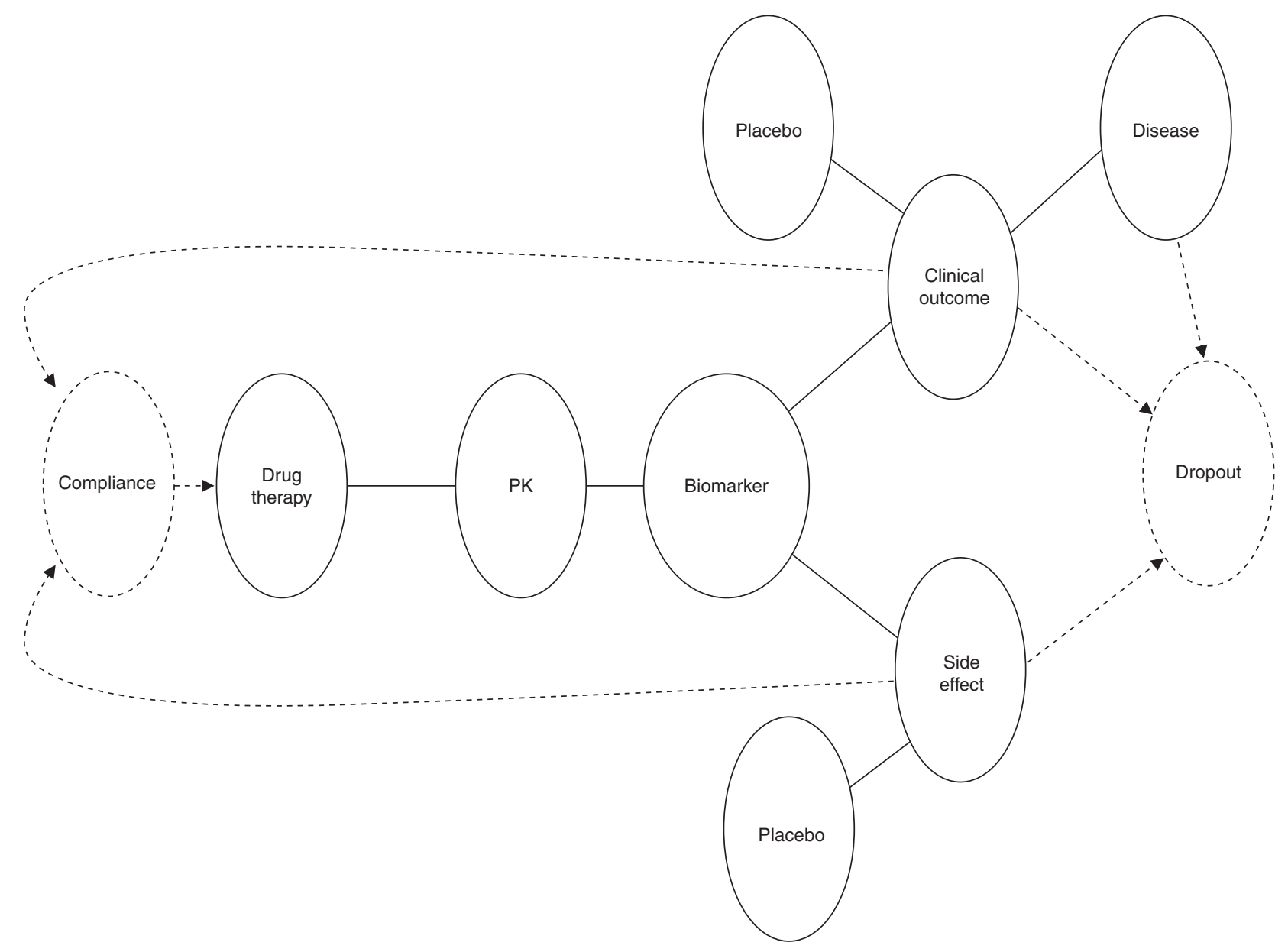

Fig. 1. Schematic representation of interactions between the drug-disease model and the execution model: the compliance model and the dropout model. Drug-disease model interactions are shown with continuous lines, while execution model interactions are shown with dashed arrows. Notice that compliance influences pharmacokinetic (PK) and pharmacodynamic models, which in turn, by feedback mechanisms, may influence compliance.

are invaluable communication tools in pharmaceutical companies.

\subsection{Proof of Concept}

In silico modelling allows the construction of complex therapeutic models integrating the quasi-totality of available knowledge on illness and treatment mechanisms. Thanks to those numerical models and CTS, one can check the likelihood of a hypothesis and so perform a proof of concept. While potential therapeutic innovations are increasingly numerous together with progress in molecular biology and genetic research, "the number of new drug and biologic applications submitted to FDA has declined significantly". ${ }^{[24]}$ On the one hand, the only way to establish the validity of a therapeutic model on which therapeutic innovation relies is the clinical trial; its experimental implemen- tation is complex, lengthy, costly and prone to randomness; on the other hand, the dangers associated with a particular metabolic profile or unwanted secondary effects can be poorly predicted by animal studies. In silico experimentation should allow pre-validation of a therapeutic model, which would be considered as a proof of concept before the decision is made to initiate the process of clinical development. Integration of the mechanisms of action of treatments and the physiopathological mechanisms of illnesses in numerical models would lead to therapeutic models that would be more reliable than discursive models.

\subsection{Optimising a Clinical Study}

Optimising a clinical study involves identifying experimental designs that maximise efficacy and minimise toxicity (best efficacy/toxicity ratio) for the lowest cost. In order to achieve 
this, CTS can play with several parameters controlling the studies: the classical parameters such as number of patients per arm or dose and dosage regimen, and the less standard such as parallel or crossover designs, number of visits per patient, choice of the clinical endpoint, choice of the comparator (placebo or some active treatment), length of the study, the influence of inclusion criteria as well as logistical and pharmacoeconomic factors. ${ }^{[25]}$

\subsection{Decision Analysis and Drug Development}

The purpose of decision analysis is to help a decision-maker think systematically about complex problems and to improve the quality of the resulting decision. One typical decision is to stop or continue drug development when a certain amount of information has been gathered (the Go/No-Go alternative). Quantitative decision-making methods may be helpful in the following situations: when decisions are difficult because the decision is complex; when there is great uncertainty about the outcome of the decision; and when the decision has multiple objectives or when different perspectives lead to different conclusions. A quantitative decision-making process can be described as following a sequence of steps: (i) identify the decision; (ii) identify alternatives; (iii) identify outcomes; (iv) model the decision; (v) evaluate the alternatives; and (vi) gain insight into all previous steps and start again if necessary. Typically, $M \& S$ will provide the tool to build the integrated exposure-response model for step four. ${ }^{[25]}$ Decision trees help by displaying the structure of a decision clearly: the sequence of decisions, the alternatives, the uncertainties, and the outcomes (figure 2).

\subsection{Test Subgroups and Complete the New Drug Application File}

CTS can also be used to estimate consequences of the treatment in subpopulations from subgroup analysis of clinical trials. The goal is to predict the benefit/risk ratio, estimated in a virtual clinical trial by resampling the existing subgroup of patients and applying to them an experimental design and therapeutic model that would take into account the specificities of those patients. However, the reliability of such extrapolations is limited and they can only be indicative.

Another purpose of CTS could be to complete the new drug application and document the as yet unstudied aspects of the new medication. One such typical example combining those two applications of CTS is provided by a prediction of outcomes in a typical subgroup of patients for docetaxel. ${ }^{[5]}$ It was shown that patients with non-small-cell lung cancer with high baseline $\alpha 1$ acid glycoprotein levels had a shorter time to disease progression and death. CTS was used to assess whether such patients might benefit from dose intensification, and showed that, if such a study were undertaken, there would be insufficient power to detect a difference. This led to the decision not to perform this subgroup study. ${ }^{[5]}$ However, once again there was a consensus by the

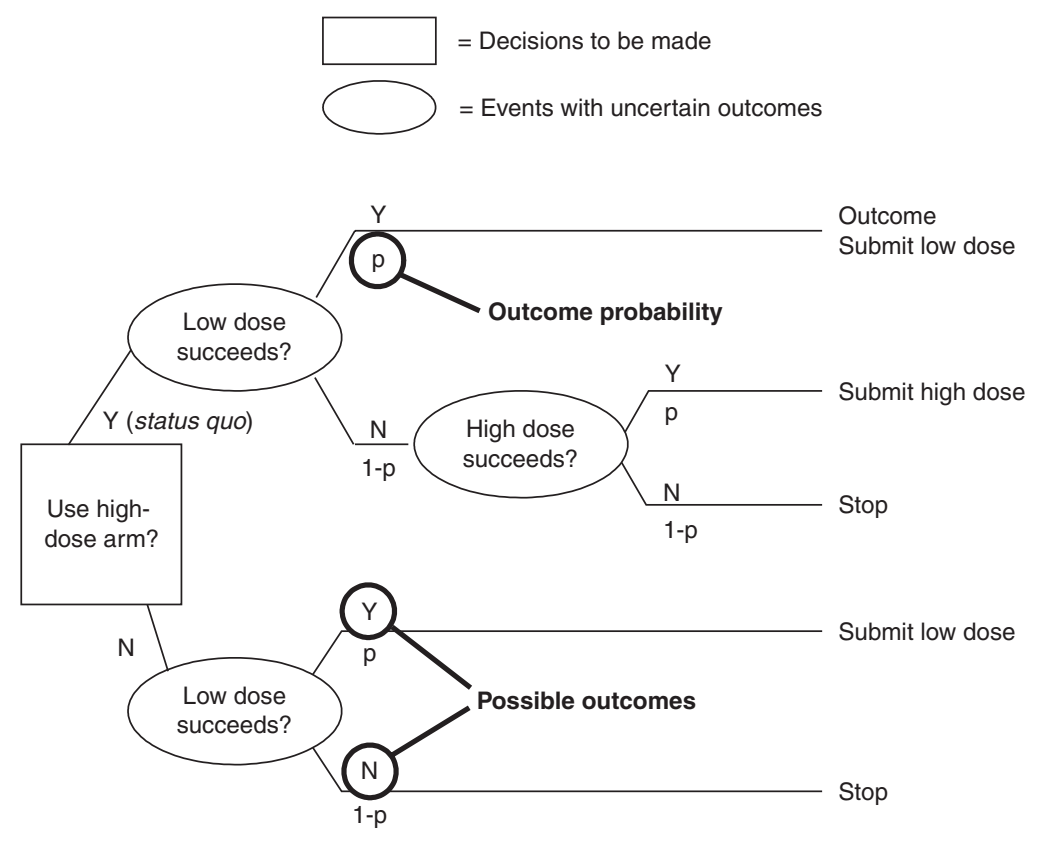

Fig. 2. Decision trees displaying the structure of a decision. $\mathbf{N}=$ no; $\mathbf{Y}=$ yes. 
round-table participants that in no way should CTS results be used as a proof to replace a real clinical trial.

\subsection{Departures from Protocol and the Execution Model}

Execution models describe protocol deviations from a specified study design. When a clinical trial is planned, it is generally envisaged that it will be executed according to a specific protocol that defines all aspects of the experimental design, from its beginning to its completion. For example, the following characteristics must be precisely defined in any clinical protocol: patient characteristics (inclusion/exclusion criteria) and number of subjects; treatments and allocation methods; dosage regimen (dose and timing of doses); measurements to be performed (type, date and time); and study duration.

Adherence to the protocol will allow estimation of the treatment outcome (safety and efficacy) with sufficient statistical power, or at least that is what is assumed. In reality, however, deviations from the protocol may lead to failure of the study to achieve its stated aims. In anticipation of protocol deviations that contribute to inflated residual variability and decreased study statistical power, trial designers tend to overpower studies in a rather arbitrary way. It should be emphasised that, in certain cases, the increase in the number of patients is not sufficient to compensate for this decrease in power, and in other cases this overpowering may result in unneeded larger studies with financial consequences for the overall drug development programme. It is difficult to estimate quantitatively the consequences of one protocol deviation on statistical study power and, a fortiori, it is almost impossible to do it for a combination of protocol deviations. The only way to study the consequences of protocol deviations is by using modelling and simulation techniques, and more specifically longitudinal stochastic models, which are the only models that can describe individual behaviours and address the impact of those protocol departures using the following 'What-if' scenarios:[26]

- what if there is noncompliance with inclusion/exclusion criteria?

- what if fewer patients than planned are accrued?

- what if the patient or clinical team does not comply with measurement times?

- what if there are missing measurements?

- what if the patient takes fewer or extra dose(s) of treatment than prescribed, but the remaining doses are taken on time?

- what if the patient stops taking the treatment but remains in the study?

- what if the patient drops out before the end of the study?

\section{Methodology}

The methodology of CTS has largely been addressed in various papers, a book and a web-published document. ${ }^{[7,22]}$ The first step in conducting a CTS is to define the objectives and the level of complexity of the model that the CTS team wants to achieve. The second step is to list assumptions, build the model and qualify it. ${ }^{[27]}$ Building the model will imply identifying the structure of the different models (PK, PD, clinical and toxic outcomes and the between- and within-patient random variability models, as well as uncertainty models. Unexplained variability is estimated to be conditional on available data using nonlinear mixed-effect models. Similarly, the precision with which model parameters are estimated will be taken into account. This precision depends first on the quantity of information available and the quality of the model (absence of model mis-specifications). For example, in the case of a perfect model, precision tends to infinity and error to zero when information quantity increases to infinity. Fortunately, the methodology of nonlinear mixed-effect models provides by default the uncertainty estimates. Ignoring this uncertainty would lead to a systematic overestimation of the therapy success rate. If no data are available to estimate part of the model or variability and uncertainty, they can be fixed to prior information, derived from previous studies or the scientific literature, or imputed after having performed a sensitivity analysis on all relevant model parameters. The next step consists of building and running the simulation, which can be split into the following: (i) writing a simulation protocol that will include various simulation study protocols; (ii) simulating 'virtual' or hybrid patients (section 2.5); (iii) creating multiple replicated simulated studies across uncertainty; (iv) representing results as distribution, tables; and (v) performing a sensitivity analysis. The validation of a simulation is performed both internally (is my simulation reproducing the observed data?) and externally by comparing it with observed data that were not used for building the different models. Most of the time, the latter validation can only be performed afterwards, since by definition CTS is used to predict the outcome of a future, not yet performed, study.

\section{Current Practices}

\subsection{Giens 2003: A Quick Survey}

A quick survey was performed among the participants of the 'Journées de Pharmacologie de Giens 2003' (46 responses/153 participants). It appeared that the M\&S approach already exists, marginally at universities, and less marginally in industry, with the main objective being the optimisation of development. The wish expressed in this poll, which has to be regarded cautiously 
given the low representativeness of the survey, is to see more development and application of this approach in the future.

\subsection{Example}

Ivabradine is a new agent that decreases heart rate and has a potential indication in stable angina pectoris. To investigate the best compromise between efficacy, safety, drug regimen, and number of patients to include in a phase III study, a CTS was conducted using a full therapeutic model. ${ }^{[6,28]}$ This example illustrates several aspects of CTS: creation of 'hybrid' patients, use of prior information from the literature for building the clinical outcome model. Briefly, a binary clinical outcome, chest pain, was simulated using a physiological model in which the coronary reserve was derived from the heart rate. Safety was defined as the absence of bradycardia. By using real data from phase I to build a PK-PD model controlling the drug effect (i.e. decrease in heart rate), and resampling heart-rate profiles from the database, 100 clinical trials were simulated, each including 100 patients in a control group and 100 receiving one oral dose (ivabradine 2.5, 5, 10,20 and $40 \mathrm{mg}$ daily or twice daily). Only $25 \%$ of the simulated trials showed a significant effect of ivabradine at doses of up to $10 \mathrm{mg}$ daily, and $48 \%$ and $55 \%$ of the trials with doses of $10 \mathrm{mg}$ twice daily and $20 \mathrm{mg}$ daily, respectively, and more than $80 \%$ of the trials with a $40 \mathrm{mg}$ daily dose. With regard to safety, $4 \%$ of patients had at least one adverse event in the untreated group, and from 5 to $13 \%$ in the treated groups for the lowest to the highest dose, respectively. The number of subjects to include in a future trial to obtain a $15 \%$ decrease in chest pain under the assumption of a $68 \%$ base risk is 239 subjects per group with $10 \mathrm{mg}$ twice daily or 196 with $20 \mathrm{mg}$ daily. Those results, which have been established using phase I data from healthy volunteers and models from the scientific literature are only indicative and need to be validated using real data.

\section{What Can Slow Down or Accelerate Implementation of M\&S}

The round table attempted to identify what conditions would allow a better integration of M\&S for drug development in the future. First, there is a need for all the actors involved in drug development and clinical pharmacology, both in the pharmaceutical industry and academia, to appropriate the methodology. For this purpose, examples showing the proof of concept need to be published, despite issues of confidentiality. Such publications are absolutely necessary to advertise the approach, make it concrete, and to provide an analysis of its attractions and limitations. The implementation of CTS implies the creation of knowledge, experimental results and specific scientific databases, and ensuring the easy access to libraries of mathematical models of biological systems.

Future acceptance of this methodology requires more resources for initiating multidisciplinary simulation teams, and the training of the actors and the teams in the multidisciplinary process, in order to organise the numerous competencies required, e.g. decompartmentalising the different areas of drug development and facilitating exchanges between them. The practical realisation of CTS could also be largely facilitated by the availability of M\&S bivalent software, allowing the creation of generic models and easy progression from model identification and validation to simulation and vice versa, i.e., from simulation to model identification with the same tool.

Another condition is the acceptance and enhanced understanding of drug agencies for such methodology. Finally, the establishment of good M\&S practice seems to be necessary. Such a draft document already exists at the Center for Drug Development Science (CDDS) in Washington and a similar work has been initiated at INSERM (Institut National de la Santé et de la Recherche Médicale), although both need to be completed and adopted. ${ }^{[22]}$

\section{Conclusion}

In various areas, such as cardiology, oncology, osteoporosis and rheumatoid arthritis, clinical trials require larger numbers of patients and a longer duration of follow-up. The risks of negative trials need to be limited as far as possible, implying that trials should be based on the soundest assumptions. The use of physiopathological and clinical trial simulations introduces a new paradigm. Now, the validation of a hypothesis is no longer confined to in vitro or in vivo experiments but can also be based on numerical and computer models, also known as in silico experiments.

\section{Participants}

J.-P. Boissel (EA643 SPC Université Lyon 1), M. Cucherat (EA643 SPC Université Lyon 1), S. Durrleman (Sanofi-Synthelabo, Chilly Mazarin), P. Girard (INSERM et EA3738 CTO Université Lyon 1), D. Guez (Laboratoires Servier, Courbevoie), R. Koen (Pharmacia SAS, St Quentin en Yvelines), C. Laveille (Laboratoires Servier, Courbevoie), H. Mathiex-Fortunet (Beaufour IPSEN Pharma, Paris), J. Micallef (CHU La Timone CPCET, Marseille), N. Missoum (Merck Sharp \& Dohme, Paris), G. Paintaud (Hôpital Bretonneau Pharmacologie, Tours), M.-C. Perault (CIC CHU, Tours), M. Tansey (Pharmacia, Jersey City, USA), J.-L. Thomas (Merck Santé, Lyon), J.-M. Treluyer (Hôpital St Vincent de Paul Pharmacologie Clinique, Paris), Ph. Variol (Pierre Fabre Médicament, Castres), T. Waegemans (L.F.B., Paris).

\section{References}

1. Brooks MM, Hallstrom A, Peckova M. A simulation study used to design the sequential monitoring plan for a clinical trial. Stat Med 1995; 14: 2227-37

2. Hale MD, Gillespie WR, Gupta SK, et al. Clinical trial simulation as a tool for increased drug development efficiency. Appl Clin Trials 1996; 5: 35-40 
3. Holford NH, Kimko HC, Monteleone JP, et al. Simulation of clinical trials. Annu Rev Pharmacol Toxicol 2000; 40: 209-34

4. Kimko HC, Reele SS, Holford NH, et al. Prediction of the outcome of a phase 3 clinical trial of an antischizophrenic agent (quetiapine fumarate) by simulation with a population pharmacokinetic and pharmacodynamic model. Clin Pharmacol Ther 2000; 68: 568-77

5. Veyrat-Follet C, Bruno R, Olivares R, et al. Clinical trial simulation of docetaxel in patients with cancer as a tool for dosage optimization. Clin Pharmacol Ther 2000; 68: 677-87

6. Chabaud S, Girard P, Nony P, et al. Clinical trial simulation using therapeutic effect modeling: application to ivabradine efficacy in patients with angina pectoris. J Pharmacokinet Pharmacodyn 2002; 29: 339-63

7. Ko HC, Dufull S. Simulation for designing clinical trials. New York, USA: Marcel Dekker, 2002

8. Bassingthwaighte JB. Strategies for the physiome project. Ann Biomed Eng 2000; 28: $1043-58$

9. Sheiner LB, Steimer JL. Pharmacokinetic/pharmacodynamic modeling in drug development. Annu Rev Pharmacol Toxicol 2000; 40: 67-95

10. What impact does the use of flight simulators have on commercial aviation? [online]. Available from URL: http://www.airlinesafety.com/faq/faq1.htm. 2004 [Accessed 2004 Jul 21]

11. Jusko WJ, Ko HC. Physiologic indirect response models characterize diverse types of pharmacodynamic effects. Clin Pharmacol Ther 1994; 56: 406-19

12. Charnick SB, Kawai R, Nedelman JR, et al. Perspectives in pharmacokinetics physiologically based pharmacokinetic modeling as a tool for drug development. J Pharmacokinet Biopharm 1995; 23: 217-29

13. Jupp DLB. Approximation to data by splines with free knots. SIAM J Numer Anal 1978; 15: 328-43

14. Wellstein A, Palm D. Correlation between pharmacological response, kinetics of plasma concentration and in vitro receptor affinities exemplified with betaadrenoceptor blocking drugs. Methods Find Exp Clin Pharmacol 1984; 6 641-4

15. Wellstein A, Palm D, Pitschner HF, et al. Receptor binding of propranolol is the missing link between plasma concentration kinetics and the effect-time course in man. Eur J Clin Pharmacol 1985; 29: 131-47

16. Jusko WJ, Ko HC, Ebling WF. Convergence of direct and indirect pharmacody namic response models. J Pharmacokinet Biopharm 1995; 23: 5-8
17. Kushler R, Brown B. A model for the identification of hormone pulses. Stat Med 1991; 10: 329-40

18. Camproux AC, Thalabard JC, Thomas G. Stochastic modeling of the hypothalamic pulse generator activity. Am J Physiol 1994; 267: E795-800

19. Hoffman A, Goldberg A. The relationship between receptor-effector unit heterogeneity and the shape of the concentration-effect profile: pharmacodynamic implications. J Pharmacokinet Biopharm 1994; 22: 449-68

20. Jusko WJ. Receptor-mediated pharmacodynamics of corticosteroids. Prog Clin Biol Res 1994; 387: 261-70

21. Troconiz IF, Sheiner LB, Verotta D. Semiparametric models for antagonistic drug interactions. J Appl Physiol 1994; 76: 2224-3

22. Holford NH, Hale MD, Ko HC, et al. Simulation in drug development: good practices. Draft Publication of the Center for Drug Development Science (CDDS). Draft version 1.0, 23 Jul, 1999 [online]. Available from URL: http://cdds.georgetown.edu/research/sddgp723.html [Accessed 2004 Jul 19]

23. Greenland S. Interpreting time-related trends in effect estimates. J Chronic Dis 1987; 40 Suppl. 2: 17S-24S

24. US Food and Drug Administration. US Department of Health and Human Services. Innovation or stagnation: challenge and opportunity on the critical path to new medical products [online]. Available from URL: http://www.fda.gov/oc/ initiatives/criticalpath/whitepaper.html [Accessed 2004 Jul 29]

25. Wada DR, Poland B. Combining drug-disease and economic modelling to inform drug development decisions. Drug Discov Today 2001; 6: 1165-70

26. Kastrissios H, Girard P. Protocol deviations and execution models. In: Ko HC, Duffull SB, editors. Simulation for designing clinical trials. New York, USA: Marcel Dekker, 2002

27. Yano Y, Beal SL, Sheiner LB. Evaluating pharmacokinetic/pharmacodynamic models using the posterior predictive check. J Pharmacokinet Pharmacodyn 2001; 28: 171-92

28. Duffull SB, Chabaud S, Nony P, et al. A pharmacokinetic simulation model for ivabradine in healthy volunteers. Eur J Pharm Sci 2000; 10: 285-94

Correspondence and offprints: Pascal Girard, INSERM et EA 3738 Ciblage Thérapeutique en Oncologie, Faculté de Médecine Lyon-Sud, BP12, 69921 Oullins Cedex, France.

E-mail: Pascal.Girard@adm.univ-lyon1.fr 